\title{
Tratamento dietético da hiper-homocisteinemia na doença arterial periférica
}

\author{
Dietary treatment of hyperhomocysteinemia in peripheral arterial \\ disease
}

Luciene de Souza Venâncio ${ }^{1}$, Roberto Carlos Burini ${ }^{2}$, Winston Bonetti Yoshida ${ }^{3}$

\begin{abstract}
Resumo
A homocisteína está envolvida na gênese da aterosclerose e, assim, é considerada um importante e prevalente fator de risco na doença arterial periférica. $\mathrm{O}$ estado nutricional vitamínico deficiente, em especial do folato, é a principal causa de hiper-homocisteinemia nesses casos. Embora ainda não haja consenso sobre a dose exata e a forma de utilização do folato em suplementos e sobre adequação alimentar ou fortificação de cereais para o tratamento da hiper-homocisteinemia, diversos estudos realizados em pacientes com doença vascular periférica mostraram que $o$ folato, isoladamente, pode reduzir as concentrações de homocisteína, bem como a concentração de alguns marcadores biológicos do processo de aterosclerose. No entanto, estudos recentes não comprovaram esse benefício sobre o processo inflamatório associado à hiper-homocisteinemia. Desta forma, embora a utilização isolada do folato seja uma terapêutica custo-efetiva no controle da hiper-homocisteinemia, seu impacto na evolução das doenças arteriais ainda persiste inconclusivo. Esta revisão abordará os efeitos obtidos com as diversas formas de utilização do folato no tratamento da hiper-homocisteinemia.
\end{abstract} lico.

Palavras-chave: Homocisteína, aterosclerose, vitamina, ácido fó-

\section{Introdução}

A doença arterial periférica (DAP) é definida como uma doença arterial das extremidades que reduz o fluxo sanguíneo durante o exercício ou, em estágios avançados, mesmo em repouso ${ }^{1}$. Estudos epidemiológicos mostram que a prevalência de DAP varia de 1,6 a $12 \%$.

As doenças do aparelho circulatório foram as principais causas de morte entre os brasileiros em 2007 $(28,2 \%)^{4}$ e na população mundial (18 milhões $)^{5}$; dentre as várias etiologias, a aterosclerose foi a causa mais comum. Entre os pacientes com doença aterosclerótica

\begin{abstract}
Homocysteine plays a role in the genesis of atherosclerosis and, thus, it is considered an important and prevalent risk factor for peripheral arterial disease. Impaired vitamin nutritional status, especially regarding folate, may be mainly attributed to hyperhomocysteinemia. Although there is still no consensus as to the exact dose and method of use of folate in supplements, dietary adjustment or cereal fortification for the treatment of hyperhomocysteinemia, several studies conducted in patients with peripheral vascular disease have shown that isolated folate may reduce homocysteine levels, as well as the levels of some biological markers in the atherosclerotic process. However, recent studies have not corroborated this benefit for the inflammatory process associated with hyperhomocysteinemia. Consequently, although the use of folate is a cost-effective therapy for the control of hyperhomocysteinemia, its impact on the evolution of vascular diseases remains inconclusive. This literature review addresses the effects of several forms of folate therapies in the treatment of hyperhomocysteinemia.
\end{abstract}

Keywords: Homocysteine, atherosclerosis, vitamin, folic acid.

sintomática, $15,9 \%$ têm doença polivascular sintomática (DAP, doença cardiovascular e cerebrovascular) ${ }^{6}$. Além disso, na fase de claudicação intermitente, cerca de $65 \%$ dos indivíduos apresentam comprometimento simultâneo e importante dos setores coronariano e cerebrovascular, e $30 \%$ dos pacientes com doença vascular coronariana ou cerebral apresentam $\mathrm{DAP}^{7}$. Desse modo, a DAP está associada a um aumento da morbimortalidade por doenças cardiovasculares (aproximadamente $30 \%$ ) e cerebrovasculares ${ }^{8}$, sendo um importante preditor das mesmas.

\footnotetext{
1. Nutricionista. Doutora em Bases Gerais da Cirurgia, Faculdade de Medicina de Botucatu (FMB), Universidade Estadual Paulista (UNESP), Botucatu, SP. Coordenadora, Curso de Graduação em Nutrição, Universidade Metodista de Piracicaba, Campus Lins, SP.

2. Professor titular, Departamento de Saúde Pública, e coordenador, Centro de Metabolismo em Exercício e Nutrição, FMB, UNESP, Botucatu, SP.

3. Professor adjunto, Departamento de Cirurgia e Ortopedia, FMB, UNESP, Botucatu, SP.
}

Apoio financeiro concedido pela Coordenação de Aperfeiçoamento de Pessoal de Nível Superior (CAPES) e pela Fundação de Amparo à Pesquisa do Estado de São Paulo (FAPESP) (processo oㅜ 03/06133-0).

Não foram declarados conflitos de interesse associados à publicação deste artigo.

Artigo submetido em 26.04.09, aceito em 03.02.10.

J Vasc Bras. 2010;9(1):28-41.

Copyright $\odot 2010$ by Sociedade Brasileira de Angiologia e de Cirurgia Vascular 
Alguns fatores de risco para o desenvolvimento da doença aterosclerótica são bastante conhecidos, como idade, sexo masculino, dislipidemia, tabagismo, hipertensão arterial sistêmica, diabetes melito, obesidade, sedentarismo e fatores genéticos ou história familiar de doença aterosclerótica ${ }^{6}$. Nos últimos anos, foram identificados outros fatores de risco, como a hiper-homocisteinemia, cujo estudo pode ampliar o entendimento sobre os mecanismos fisiopatológicos da aterosclerose e possibilitar o desenvolvimento de novas medidas preventivas ou terapêticas.

\section{Metabolismo da homocisteína e hiper-homocisteinemia na DAP}

A homocisteína é um aminoácido sulfurado não protéico; não é um constituinte da dieta e não é formador de proteínas, mas é produzido exclusivamente como um produto intermediário do metabolismo intracelular do aminoácido essencial metionina ${ }^{9,10}$ (Figura 1).

$$
\text { HS- }-\mathrm{CH}_{2}-\mathrm{CH}_{2}-\underset{\mathrm{NH}_{2}}{\mathrm{C}}-\mathrm{COOH}
$$

Figura 1 - Representação da molécula de homocisteína ${ }^{11}$

O metabolismo intracelular da homocisteína ocorre por meio de duas vias de remetilação, responsáveis pela conversão da homocisteína em metionina, e uma via de transulfuração, que converte a homocisteína em cisteína (Figura 2). Essas vias são dependentes das vitaminas B6, B12 e folato, que atuam como coenzimas ou cossubstratos. Em uma das vias de remetilação, o doador do grupo metil é

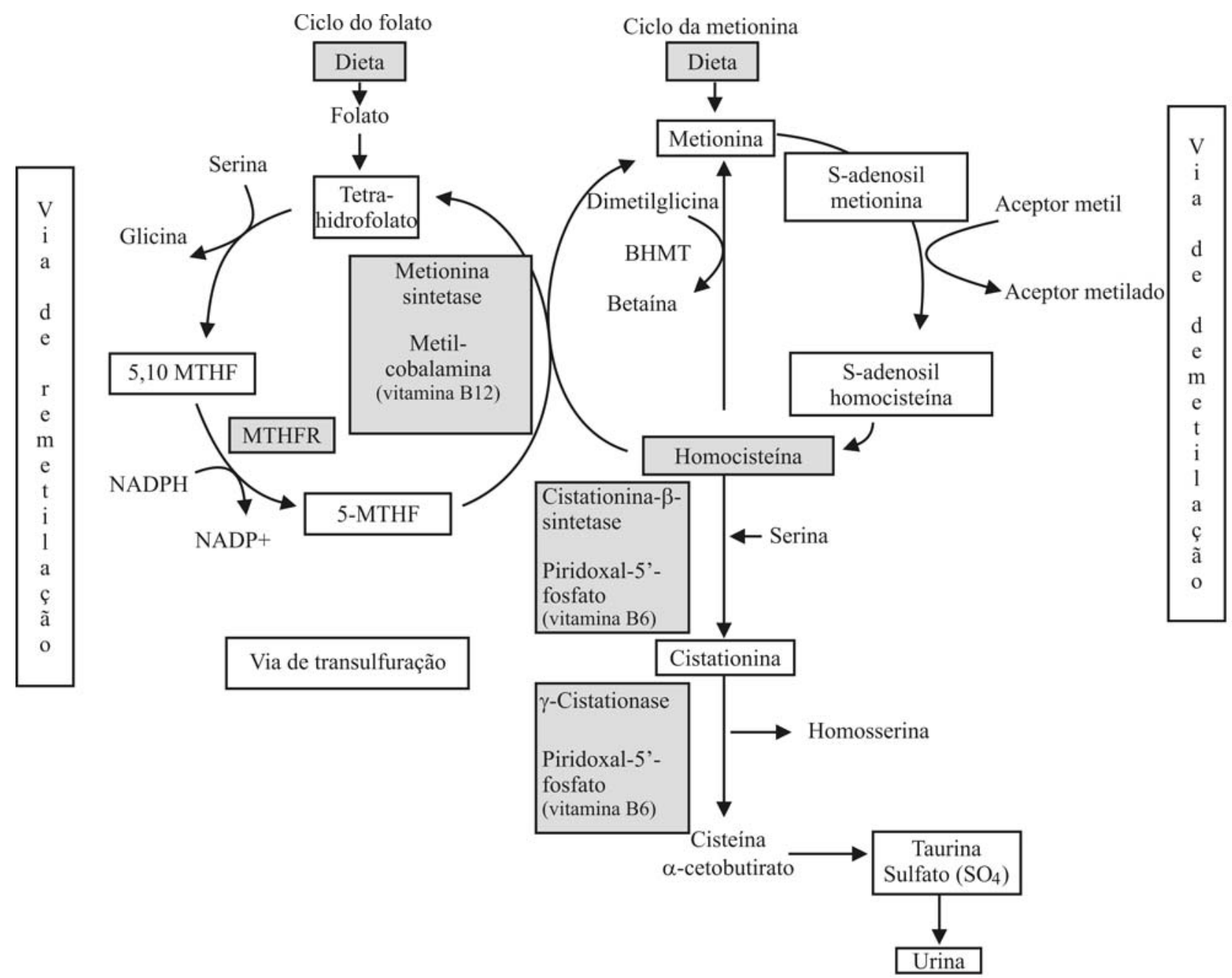

BHMT $=$ betaína-homocisteína metiltransferase; $\mathrm{MTHF}=$ metilenotetrahidrofolato; MTHFR = metilenotetrahidrofolato redutase; $\mathrm{NADPH}=$ nicotinamida adenina dinucleotídeo fosfato na forma reduzida; NADP+ nicotinamida adenina dinucleotídeo fosfato.

Figura 2 - Representação esquemática das vias de metabolização da homocisteína ${ }^{12}$ 
o 5-metilenotetrahidrofolato (5-MTHF), forma principal do folato no plasma, produzido a partir da redução do 5,10-metilenotetrahidrofolato pela enzima metilenotetrahidrofolato redutase (MTHFR). A transferência do grupo metil para a homocisteína é feita mediante ação da enzima metionina-sintetase (MS), que tem como cofator a metilcobalamina (MCOB), uma coenzima derivada da cobalamina (vitamina B12). Subsequentemente, uma nova molécula de 5-MTHF é sintetizada pela transferência de um átomo de carbono (de uma fonte de carbono como serina) para uma molécula de tetrahidrofolato, produzindo metilenotetrahidrofolato e glicina ${ }^{13}$.

$\mathrm{Na}$ outra via de remetilação (demetilação), que acontece principalmente no fígado, o doador do grupo metil para a conversão da homocisteína em metionina é a betaína. Uma vez formada, a metionina é ativada pelo adenosina trifosfato (ATP), formando S-adenosil-metionina, que funciona como doador de grupo metil para uma diversidade de receptores. O produto dessas reações de metilação é a S-adenosil-homocisteína, a qual é hidrolisada regenerando a homocisteína, que se torna disponível para iniciar um novo ciclo de transferência de grupos metil ${ }^{14}$.

Na via de transulfuração, inicialmente a homocisteína se condensa com uma molécula de serina para formar cistationina em reação irreversível que é catalisada pela enzima cistationina- $\beta$-sintetase (CBS) e que tem como cofator o piridoxal-5'-fosfato, um derivado da piridoxina ou vitamina B6. Posteriormente, a cistationina é hidrolisada em cisteína e ácido $\alpha$-cetobutirato, reação esta que também requer vitamina B6 e é catalisada pela enzima $\gamma$-cistationase. Desta forma, além da produção de cisteína, a via de transulfuração é responsável pelo catabolismo da homocisteína tóxica, que não é necessária no ciclo de transferência de grupos metil. Portanto, essa via de metabolização visa a excreção renal da homocisteína, diminuindo a concentração sérica desse aminoácido. Em condições metabólicas normais, a metabolização da homocisteína é distribuída igualmente entre essas duas vias, que são coordenadas e competem pela utilização da homocisteína disponível ${ }^{14}$.

A concentração normal de homocisteína no plasma quando analisada em jejum é de aproximadamente $10 \mu \mathrm{mol} / \mathrm{L}$ devido ao mecanismo de exportação celular, com variação entre 5 e $15 \mu \mathrm{mol} / \mathrm{L}$; acima desses valores caracteriza-se a hiper-homocisteinemia ${ }^{15}$. Kang et al. ${ }^{16}$ classificaram arbi- trariamente a hiper-homocisteinemia nas formas grave, para concentrações maiores que $100 \mu \mathrm{mol} / \mathrm{L}$, moderada, para concentrações entre 31 e $100 \mu \mathrm{mol} / \mathrm{L}$, e leve, para concentrações entre 15 e $30 \mu \mathrm{mol} / \mathrm{L}$.

Desde a descoberta da homocisteína, diversos e importantes estudos prospectivos e caso-controle mostraram que a hiper-homocisteinemia é um fator de risco para doenças vasculares e é prevalente em pacientes com DAP (28 a $60 \%^{17,18}$, comparado com $1 \%$ na população geral $)^{7}$. As concentrações médias de homocisteína em pacientes com diversas manifestações de DAP, como claudicação intermitente $^{19}$, lesões íleo-femorais ${ }^{20}$, síndrome de Leriche $^{21}$, estenose de carótida ${ }^{22}$ e aneurisma de aorta abdominal ${ }^{23}$, mostraram-se significantemente superiores aos encontrados nos controles. Aproximadamente $30 \%$ dos pacientes jovens com DAP apresentam hiper-homocisteinemia. Estudos nacionais em pacientes com doença arterial coronariana $^{24-26}$ e arterial periférica ${ }^{18,27,28}$ confirmaram a alta prevalência de hiper-homocisteinemia (20 a 60\%) e a concentração elevada de homocisteína nos doentes em relação aos controles. Um estudo de meta-análise com amostra de aproximadamente 4.000 pessoas concluiu que um aumento de $5 \mu \mathrm{mol} / \mathrm{L}$ nas concentrações plasmáticas de homocisteína está associado a desenvolvimento de doença cardiovascular na razão de risco (odds ratio) de 1,6 para homens e 1,8 para mulheres, comparado a 6,8 para pacientes com $\mathrm{DAP}^{17}$. De acordo com as evidências atuais existentes, a hiper-homocisteinemia apresenta razão de risco de 3,0 para pacientes com DAP sintomática ${ }^{7}$. Além disso, estudos prospectivos evidenciaram que a hiper-homocisteinemia está associada a aumento do risco de morte precoce por doença cardiovascular ${ }^{29}$ e progressão da $\mathrm{DAP}^{30,31} \mathrm{e} \mathrm{da}$ doença arterial coronariana não-fatal em pacientes com DAP sintomática ${ }^{32}$.

As causas mais comuns de hiper-homocisteinemia na população em geral estão relacionadas aos defeitos genéticos na codificação de enzimas ou à defíciência nutricional de vitaminas que estão envolvidas no metabolismo da homocisteína. A concentração plasmática de homocisteína está inversamente associada às concentrações sanguíneas de folato, vitamina B6 e B12 e à ingestão dessas vitaminas, principalmente em idosos ${ }^{33}$.

A hiper-homocisteinemia genética é frequentemente resultado de alterações moleculares associadas a heterozi- 
gose da MTHFR ou CBS. Estima-se que 5 a $10 \%$ dos casos de hiper-homocisteinemia grave são causados por defeitos nas vias de remetilação, sendo a deficiência homozigótica de MTHFR (677CT) mais comum e menos grave quando comparada à causada pela deficiência de CBS, porém com pior prognóstico, porque não responde bem aos tratamentos disponíveis ${ }^{34}$. Indivíduos homozigotos mutantes para a enzima MTHFR apresentam apenas 30\% da atividade observada nos indivíduos com genótipo normal, valor este que é de até $65 \%$ para o genótipo heterozigoto ${ }^{35}$. A homozigose para a enzima MTHFR está presente em 5 a 20\% da população geral e foi verificada em $16,7 \%$ dos pacientes com DAP, os quais tinham homocisteína moderadamente aumentada $^{36}$. No Brasil, foi verificado que 19\% de 191 indivíduos com doença arterial coronariana eram homozigotos para a variante termolábil da enzima MTHFR, mas nenhuma correlação com a homocisteinemia foi estuda$\mathrm{da}^{37}$. Elevações moderadas na concentração de homocisteína não são encontradas em todos os portadores desse polimorfismo, o que sugere que o fenótipo deve ser influenciado por outros fatores, como estado nutricional vitamínico, hormônios, algumas doenças e medicamentos ${ }^{38}$. Existe grande influência das concentrações séricas de folato no polimorfismo da enzima MTHFR, e a hiper-homocisteinemia em indivíduos homozigotos só é observada quando existe deficiência de folato (valores inferiores a $15,4 \mu \mathrm{mol} / \mathrm{L})^{39}$.

\section{Mecanismos patogênicos da hiper-homocisteinemia}

Apesar da grande quantidade de dados epidemiológicos estabelecendo a correlação entre hiper-homocisteinemia e aumento do risco para doenças vasculares, os mecanismos pelos quais a hiper-homocisteinemia contribui para a aterogênese e trombogênese são hipotéticos e controversos. A agressão ao endotélio parece ser um dos mecanismos pelos quais a homocisteína leva à lesão vascular. Trabalhos pioneiros ${ }^{40,41}$ com primatas não humanos (babuínos) mostraram que a injeção intravenosa de L-homocisteína durante 5 dias provocou lesão endotelial caracterizada pela descamação endotelial, proliferação de células musculares lisas e espessamento da camada íntima vascular, mediada pela redução da meia-vida plaquetária, com a rápida formação de lesões vasculares, similares a lesões ateroscleróticas precoces em humanos. O grau de lesão endotelial provocada pela hiper-homocisteinemia era semelhante ao observado em associação com outros fato- res de risco, como na hipercolesterolemia e na hipertensão arterial sistêmica.

Pesquisas experimentais nacionais e recentes reforçaram o papel da hiper-homocisteinemia induzida pela sobrecarga de metionina na formação de placa aterosclerótica de aorta em suínos ${ }^{42}$ e artéria ilíaca em coelhos ${ }^{43}$. Após período de 30 ou 60 dias de dieta rica em metionina, houve aumento significante nas concentrações de homocisteína e na formação de placas ateroscleróticas por células espumosas, mas não foram observadas células musculares lisas, cristais de colesterol ou células inflamatórias.

Um dos principais mecanismos de disfunção endotelial induzida pela hiper-homocisteinemia estaria relacionado à diminuição da biodisponibilidade do fator de relaxamento do endotélio, o óxido nítrico, sintetizado a partir de L-arginina pela ação da enzima óxido nítrico-sintase. $\mathrm{O}$ óxido nítrico é um potente vasodilatador endógeno, que inibe a agregação plaquetária, a migração de leucócitos e a proliferação e migração da célula muscular lisa e restringe a ativação e expressão de moléculas de adesão e a produção de ânions superóxido ${ }^{44}$. Estudos prévios em animais hiper-homocisteinêmicos mostraram que a homocisteína reduziu a biodisponibilidade do óxido nítrico em cultura de células endoteliais, provavelmente por indução do estresse oxidativo, que inativa o óxido nítrico, ou por inibição da síntese da óxido nítrico-sintase, ou ainda por aumentar as concentrações de dimetilarginina assimétrica, que contribui para a redução da biobisponibilidade do óxido nítrico ${ }^{45}$.

Especula-se, também, que a hiper-homocisteinemia exerceria um papel importante na disfunção endotelial através de mecanismos oxidativos e inflamatórios. O grupo sulfidrila (SH), presente na homocisteína, é altamente reativo por ser doador de elétrons nos sistemas de oxidação, sendo oxidado rapidamente em dissulfeto (SS). A homocisteína é prontamente oxidada quando chega ao plasma, principalmente em consequência de sua auto-oxidação para formar SS, incluindo a homocistina, SS derivados de homocisteína e a homocisteína tiolactona, sendo esta última a forma mais reativa da homocisteína. $\mathrm{O}$ grupo $\mathrm{SH}$ dos SS e da homocisteína tiolactona reagiria com o oxigênio, produzindo o peróxido de hidrogênio e o superóxido, os quais iniciariam a peroxidação lipídica tanto na superfície endotelial como nas lipoproteínas de baixa densidade $(\mathrm{LDL})^{46}$. A oxidação da LDL, favorecida principalmente 
pela homocisteína tiolactona, formaria agregados que seriam captados por macrófagos na camada íntima das artérias, os quais estimulariam uma resposta pró-inflamatória vascular por meio da expressão de moléculas de adesão, proteínas quimiotáticas e fatores de crescimento para formar as células espumosas e, consequentemente, as lesões ateromatosas. Além disso, a auto-oxidação da homocisteína no plasma poderia favorecer redução na expressão e atividade da glutationa peroxidase e, assim, inibir o potencial antioxidante das células endoteliais ${ }^{47}$. Em culturas de células endoteliais, células musculares lisas e monócitos humanos, a hiper-homocisteinemia induziu a expressão da proteína quimiotática de monócitos (monocyte chemoattractant protein, 1-MCP-1) e da interleucina-8 (IL-8) por ativação do fator nuclear kappa B (nuclear factor kappa B, $\mathrm{NF}-\mathrm{kB}$ ) e indução da proteína $\mathrm{C}$ reativa (PCR) de fase aguda positiva. Essas substâncias ampliariam a resposta inflamatória vascular e contribuiriam para o início e a progressão da aterosclesorose ${ }^{48}$.

Outros efeitos da homocisteína seriam alterações nas propriedades antitrombóticas do endotélio vascular. Estudos in vitro em células expostas à homocisteína demonstraram um aumento da atividade dos fatores de coagulação XII e V, redução da ativação da proteína $\mathrm{C}$, inibição do ativador de plasminogênio tecidual, redução da biodisponibilidade do óxido nítrico e prostaciclina, estimulação da agregação plaquetária, aumento da atividade do fator de von Willebrand, inibição da expressão da trombomodulina, indução da expressão do fator tecidual e supressão da expressão do heparan-sulfato na parede vascular ${ }^{49}$. Todas essas alterações gerariam um ambiente trombogênico vascular, com a ativação da cascata de coagulação e modificação do tônus vascular.

A variabilidade de ações da homocisteína apontada por esses estudos demonstra que ainda não há uma hipótese única explicativa para os efeitos aterotrombogênicos da homocisteína.

\section{Tratamento nutricional da hiper-homocisteinemia}

Fatores nutricionais, em particular o consumo e a concentração sérica de folato, vitamina B12 e B6, parecem ser os parâmetros mais importantes na metabolização da homocisteína. As deficiências, isoladas ou combinadas, das vitaminas envolvidas nas diversas vias do metabolismo da homocisteína seriam importantes marcadores de hiper-ho- mocisteinemia ${ }^{50}$. Em idosos norte-americanos, dois terços (67\%) dos casos de hiper-homocisteinemia foram atribuídos a hábitos dietéticos inadequados em relação a uma ou mais vitaminas do complexo $\mathrm{B}^{51}$. Em indivíduos portadores de DAP em nosso meio, a frequência de hiper-homocisteinemia naqueles com ingestão insuficiente de duas ou três vitaminas do complexo B $(52,5 \%)$ foi significantemente maior em relação a aqueles com ingestão insuficiente de uma vitamina $(7,5 \%)$, o que confirma a relação entre hiper-homocisteinemia e estado nutricional vitamínico ${ }^{18}$. De acordo com estudo prospectivo de 12 anos de seguimento realizado com 46.036 participantes do sexo masculino $^{52}$, o consumo de folato alimentar e suplementos contendo ácido fólico estariam associados inversamente com a ocorrência de DAP e poderiam contribuir para a prevenção desta.

A deficiência dietética de folato provocaria formação insuficiente de 5-MTHF, a qual é necessária como grupo doador de radical metil na remetilação da homocisteína para metionina. A deficiência da vitamina B12, por sua vez, levaria a um prejuízo na transferência do radical metil da 5-MTHF para a homocisteína na via de remetilação através da metionina-sintetase. Logo, a deficiência da vitamina B6 prejudicaria a conversão da homocisteína em cisteína pelas enzimas CBS e $\gamma$-cistationase, as quais são ativadas pela vitamina B6 na via da transulfuração. Essas condições favoreceriam o acúmulo de homocisteína intracelular, a qual seria transportada para o compartimento extracelular e, consequentemente, provocaria aumento das concentrações circulantes de homocisteína. Esse processo restringiria a toxicidade intracelular, mas, por outro lado, poderia expor o meio vascular aos efeitos deletérios do excesso de homocisteína ${ }^{53}$. Dentre essas vitaminas, o folato seria o determinante dietético mais importante da concentração de homocisteína, pois sua deficiência poderia restringir a via de remetilação da homocisteína para metionina e favorecer o seu acúmulo no meio extracelular ${ }^{50}$.

Folato é um termo genérico utilizado para denominar essa vitamina essencial e hidrossolúvel do complexo B, que se apresenta sob a forma ativa de ácido tetrahidrofólico e tem função de coenzima para reações de transferência de unidades de carbono necessárias em várias vias metabólicas, incluindo o metabolismo de purinas e pirimidinas, bem como interconversões de aminoácidos. O ácido fólico (ácido pteroilmonoglutamato), forma oxidada e estável do 
folato, embora seja raramente encontrado nos alimentos, é a forma utilizada em suplementos vitamínicos e produtos alimentícios fortificados, representando $20 \%$ do folato da dieta. Nos alimentos naturais, o chamado folato alimentar é encontrado na forma de pteroilpoliglutamato, que representa aproximadamente $80 \%$ do folato da dieta (Tabela 1 ).

Tabela 1 - Conteúdo de folato, vitamina B12 e B6 em diversos $\operatorname{alimentos}^{54}$

\begin{tabular}{|c|c|}
\hline Alimento & Conteúdo \\
\hline \multicolumn{2}{|l|}{ Folato $(\mu \mathrm{g} / 100 \mathrm{~g})$} \\
\hline Fígado de galinha grelhado & 560 \\
\hline Farinha de soja & 303 \\
\hline Fígado bovino grelhado & 253 \\
\hline Feijão cozido & 149 \\
\hline Espinafre cozido & 73 \\
\hline Brócolis cru & 71 \\
\hline Farinha de aveia & 52 \\
\hline Farelo de trigo & 44 \\
\hline Ovo cozido & 44 \\
\hline Laranja & 30 \\
\hline Couve crua & 29 \\
\hline Pão branco & 25 \\
\hline Banana & 20 \\
\hline Tomate cru & 15 \\
\hline Batata cozida & 9 \\
\hline \multicolumn{2}{|l|}{ Vitamina B12 $(\mu \mathrm{g} / 100 \mathrm{~g})$} \\
\hline Fígado bovino grelhado & 70,5 \\
\hline Fígado de galinha grelhado & 16,8 \\
\hline Peixe grelhado & 2,8 \\
\hline Carne bovina & 2,4 \\
\hline Queijo tipo mussarela & 2,2 \\
\hline Camarão cozido & 1,8 \\
\hline Ovo cozido & 1,3 \\
\hline Leite de vaca integral & 0,4 \\
\hline \multicolumn{2}{|l|}{ Vitamina B6 (mg/100 g) } \\
\hline Fígado bovino grelhado & 1,00 \\
\hline Fígado de galinha grelhado & 0,87 \\
\hline Banana & 0,36 \\
\hline Farelo de trigo & 0,34 \\
\hline Oleaginosas & 0,27 \\
\hline Batata cozida & 0,26 \\
\hline Abacate & 0,25 \\
\hline Brócolis cozido & 0,20 \\
\hline Farinha de aveia & 0,16 \\
\hline Feijão cozido & 0,06 \\
\hline
\end{tabular}

O consumo ideal das vitaminas B12, B6 e folato é definido pela Recommended Dietary Allowances (RDA) ou Recomendações de Cotas Alimentares preconizadas pelas Dietary Reference Intakes ${ }^{55}$, definidas como nível de consumo alimentar suficiente para satisfazer as necessidades de quase todo indivíduo saudável (entre 97 e 98\%) compreendido num determinado grupo, faixa etária e estágio da vida (Tabela 2 ).

Em 1996, a Food and Drug Administration (FDA) regulamentou a fortificação de cereais como farinha, arroz, massas e milho com $140 \mu \mathrm{g}$ de ácido fólico por $100 \mathrm{~g}$ de produto com o objetivo de reduzir o risco de defeitos do tubo neural em recém-nascidos. Após essa regulamentação, foi observado um aumento significante nas concentrações de folato sérico e redução significante nas concentrações de homocisteína em 350 adultos e idosos participantes do Framingham Offspring Study ${ }^{56}$. A fortificação com 499 e $665 \mu \mathrm{g}$ de ácido fólico para $30 \mathrm{~g}$ de cereais matinais foi igualmente eficaz em reduzir a homocisteinemia e aumentar o folato sérico em pacientes com doença arterial coronariana. Entretanto, a fortificação com $127 \mu \mathrm{g}$, quantidade que se aproxima daquela proposta pela FDA, foi insuficiente para reduzir significantemente a homocisteinemia e induzir um aumento moderado de folato sérico. Portanto, a homocisteinemia reduziu e o folato sérico aumentou linearmente com o aumento de ácido fólico contido no cereal. Diante desses resultados, os autores sugeriram que um nível de fortificação superior (aproximadamente $350 \mu \mathrm{g}$ ) ao proposto pela FDA deveria ser

Tabela 2 - Recomendações de cotas alimentares (Recommended Dietary Allowances, RDA) preconizadas pela Dietary Reference Intakes ${ }^{55}$

\begin{tabular}{lccc}
\hline $\begin{array}{l}\text { Sexo/faixa } \\
\text { etária (anos) }\end{array}$ & $\begin{array}{c}\text { Folato } \\
(\mu \mathrm{g} / \mathrm{dia})\end{array}$ & $\begin{array}{c}\text { Vitamina B6 } \\
(\mathrm{mg} / \mathrm{dia})\end{array}$ & $\begin{array}{c}\text { Vitamina B12 } \\
(\mu \mathrm{g} / \mathrm{dia})\end{array}$ \\
\hline $\begin{array}{l}\text { Homens } \\
19-30\end{array}$ & 400 & 1,3 & 2,4 \\
$31-50$ & 400 & 1,3 & 2,4 \\
$51-70$ & 400 & 1,7 & 2,4 \\
71 ou mais & 400 & 1,7 & 2,4 \\
Mulheres & & & \\
$19-30$ & 400 & 1,3 & 2,4 \\
$31-50$ & 400 & 1,3 & 2,4 \\
$51-70$ & 400 & 1,5 & 2,4 \\
71 ou mais & 400 & 1,5 & 2,4 \\
\hline
\end{tabular}


assegurado $^{57}$. O Brasil não dispõe de informações recentes, de representatividade nacional, sobre carências de micronutrientes em adultos. Mesmo assim, houve decisão governamental sobre a fortificação universal das farinhas de trigo e milho produzidas no País com ácido fólico ${ }^{58}$, com base em estudos de abrangência local realizados por diferentes instituições em várias regiões geográficas. A resolução tornou obrigatória a fortificação de cereais como farinha de trigo e milho com $150 \mu \mathrm{g}$ de ácido fólico por $100 \mathrm{~g}$ de cereal, o que representa $37 \%$ da RDA para adultos, com o objetivo de prevenir defeitos do tubo neural em recém-nascidos e carência de folato em crianças e adultos 59 .

Considerando-se que a hiper-homocisteinemia é comum em indivíduos que apresentam comprometimento nutricional das vitaminas $\mathrm{B} 6, \mathrm{~B} 12 \mathrm{e}$, principalmente, do folato, e pela significativa participação dessas vitaminas na homocisteinemia, diversos estudos utilizaram com sucesso essas vitaminas para reduzir as concentrações sanguíneas desse aminoácido. $\mathrm{O}$ folato, isoladamente ou em combinação com as vitaminas B6 e B12, pode reduzir a homocisteína plasmática, inclusive na ausência de deficiências. Ainda não há consenso sobre a dose exata dessas vitaminas para o tratamento da hiper-homocisteinemia, sendo que diferentes doses foram utilizadas em muitos estudos. A contribuição da terapia vitamínica, em especial do ácido fólico, na hiper-homocisteinemia é muito estudada em indivíduos saudáveis e na doença arterial coronariana, mas ainda é escassa na DAP (Tabela 3).

Meta-análise de 11 estudos realizada por Boushey et al. ${ }^{17}$ em 1995 mostrou resultados pioneiros do efeito do tratamento com ácido fólico sobre a homocisteinemia nas doenças vasculares coronariana, cerebral e periférica. Nove estudos de intervenção mostraram redução da homocisteína e normalização do folato sérico após suplementação isolada de ácido fólico (mais que $400 \mu \mathrm{g} / \mathrm{dia}$ ) e concluíram que o consumo de aproximadamente $200 \mu \mathrm{g}$ de folato dietético (três porções ou mais de frutas e vegetais ao dia) reduziria em $4 \mu \mathrm{mol} / \mathrm{L}$ as concentrações de homocisteína na doença cardiovascular, cerebrovascular e arterial periférica. Entretanto, os autores ressaltaram que nem todas as doenças vasculares associadas à hiper-homocisteinemia seriam prevenidas com a suplementação de ácido fólico e que a fortificação ou aumento do consumo de alimentos com folato poderia ter um impacto preventivo maior.
Em outra meta-análise com 12 estudos randomizados e 1.114 indivíduos $^{60}$, os efeitos redutores do ácido fólico sobre a homocisteína na doença vascular também foram verificados. A utilização diária de 50 a $500 \mu \mathrm{g}$ de ácido fólico entre 3 e 12 semanas reduziu em $25 \%$ a concentração de homocisteína, e a redução foi maior em indivíduos hiper-homocisteinêmicos $(>12 \mu \mathrm{mol} / \mathrm{L})$ e com deficiência sérica de folato $(<12 \mu \mathrm{mol} / \mathrm{L})$ antes do tratamento com ácido fólico. Os autores relataram ainda que a suplementação com vitamina B12 potencializou a redução da homocisteína em $7 \%$ e que a vitamina $\mathrm{B} 6$ não teve efeito adicional na redução da homocisteína.

Estudos realizados em nosso meio confirmaram esses achados. Um estudo caso-controle investigou suplementação de ácido fólico $(500 \mu \mathrm{g})$ associado à vitamina B12 (40 mg) em 40 pacientes com DAP sintomática e mostrou eficiência na normalização da homocisteinemia em 4 semanas de tratamento (antes, 18,80 $\pm 7,73 \mu \mathrm{mol} / \mathrm{L}$; após, $11,34 \mu \mathrm{mol} / \mathrm{L} ; \mathrm{p}<0,001)$, ao contrário da suplementação com vitamina B6 (250 mg), que não alterou a homocisteinemia $^{64}$. Da mesma forma, outro estudo do tipo cruzado realizado com indivíduos adultos hipertensos praticantes de exercício físico supervisionado mostrou redução significante das concentrações médias de homocisteína após 2 meses de tratamento com ácido fólico $(500 \mu \mathrm{g})$ na ausência do uso de diurético ${ }^{65}$.

Um estudo comparativo do efeito de três formas de suplementação com ácido fólico sobre a homocisteinemia mostraram que: 1) o consumo diário de cereais matinais fortificados e 2) o uso de suplementos pareceram ser os métodos mais eficientes para aumentar os níveis de folato e reduzir a homocisteína sanguínea; 3) o aumento no consumo de alimentos ricos em folato não seria, propriamente, uma estratégia eficiente para reduzir a homocisteinemia, provavelmente pelas variações na biodisponibilidade do folato em frutas e vegetais e pela ingestão pouco frequente desses alimentos ${ }^{62}$. Outros autores sugeriram ser improvável que a dieta isoladamente fosse suficiente para aumentar a concentração de folato circulante e reduzir a homocisteinemia, uma vez que o folato alimentar (pteroilpoliglutamato) apresentaria metade da biodisponibilidade do ácido fólico proveniente de suplementos (pteroilmonoglutamato ${ }^{66}$. A maior parte dos folatos presentes nos alimentos, embora bastante estável à luz, é lábil e termossensível. Essas características levam a perdas conside- 
Tabela 3 - Resultados obtidos em estudos de intervenção para redução da concentração de homocisteína plasmática

\begin{tabular}{|c|c|c|c|c|c|}
\hline $\begin{array}{l}\text { Autor (desenho do } \\
\text { estudo) }\end{array}$ & $\begin{array}{l}\text { População } \\
\text { estudada }\end{array}$ & $\begin{array}{l}\text { Número de } \\
\text { pacientes }\end{array}$ & $\begin{array}{c}\text { Período do } \\
\text { tratamento (semanas) }\end{array}$ & Tratamento & Resultados \\
\hline $\begin{array}{l}\text { Boushey et al. }{ }^{17} \\
\text { (meta-análise) }\end{array}$ & $\begin{array}{l}\text { Coronariana } \\
\text { Cerebrovascular } \\
\text { Arterial periférica }\end{array}$ & - & - & $>400 \mu \mathrm{g}$ de ácido fólico & $\downarrow 4 \mu \mathrm{mol} / \mathrm{L}$ de Hct \\
\hline \multirow{3}{*}{$\begin{array}{l}\text { Homocysteine Lowering } \\
\text { Trialists' Collabora- } \\
\text { tion }^{60} \text { (meta-análise) }\end{array}$} & & 1.114 & 3 e 12 & $\begin{array}{l}50-500 \mu \mathrm{g} \text { de ácido } \\
\text { fólico }\end{array}$ & $\downarrow 25 \% \mathrm{Hct}$ \\
\hline & & & & $0,5 \mathrm{mg}$ de vitamina B12 & $\downarrow$ adicional de $7 \%$ Hct \\
\hline & & & & $16,5 \mathrm{mg}$ de vitamina $\mathrm{B} 6$ & Sem alteração \\
\hline \multirow[t]{3}{*}{$\begin{array}{l}\text { Malinow et al. } \\
\text { (duplo-cego cruzado) }\end{array}$} & $\begin{array}{l}\text { Arterial } \\
\text { coronariana }\end{array}$ & 75 & 5 & $\begin{array}{l}\text { Fortificação de cereais } \\
127 \mu \mathrm{g} / 30 \mathrm{~g}\end{array}$ & $\begin{array}{l}\downarrow 3,7 \% \mathrm{Hct} \\
(\mathrm{p}=0,24)\end{array}$ \\
\hline & & & & $499 \mu \mathrm{g} / 30 \mathrm{~g}$ & $\begin{array}{l}\downarrow 11 \% \text { Hct } \\
(\mathrm{p}<0,001)\end{array}$ \\
\hline & & & & $665 \mu \mathrm{g} / 30 \mathrm{~g}$ & $\begin{array}{l}\downarrow 14 \% \mathrm{Hct} \\
(\mathrm{p}=0,001)\end{array}$ \\
\hline \multirow[t]{3}{*}{$\begin{array}{l}\text { Brouwer et al. }^{61} \\
\text { (caso-controle) }\end{array}$} & Adultos saudáveis & 66 & 4 & $\begin{array}{l}560 \mu \mathrm{g} \text { de folato } \\
\text { alimentar }\end{array}$ & $\begin{array}{l}\downarrow 11,0 \text { para } 9,5 \mu \mathrm{mol} / \mathrm{L} \\
\text { de Hct } \\
(\mathrm{p}<0,001)\end{array}$ \\
\hline & & & & $\begin{array}{l}210 \mu \mathrm{g} \text { folato alimentar } \\
+500 \mu \mathrm{g} \text { suplemento de } \\
\text { ácido fólico }\end{array}$ & $\begin{array}{l}\downarrow 10,8 \text { para } 9,0 \mu \mathrm{mol} / \mathrm{L} \\
\text { de Hct } \\
(\mathrm{p}<0,001)\end{array}$ \\
\hline & & & & $\begin{array}{l}250 \mu \mathrm{g} \text { suplemento de } \\
\text { ácido fólico }\end{array}$ & Sem alteração \\
\hline \multirow[t]{3}{*}{$\begin{array}{l}\text { Riddell et al. } \\
\text { (caso-controle) }\end{array}$} & $\begin{array}{l}\text { Adultos e idosos } \\
\text { saudáveis }\end{array}$ & 65 & 12 & $\begin{array}{l}\text { Ácido fólico } 437 \mu \mathrm{g} \\
\text { suplemento }\end{array}$ & $\begin{array}{l}\downarrow 21 \% \text { da Hct } \\
(\mathrm{p}<0,001)\end{array}$ \\
\hline & & & & $\begin{array}{l}298 \mu \mathrm{g} \text { cereal } \\
\text { fortificado }\end{array}$ & $\begin{array}{l}\downarrow 24 \% \text { da Hct } \\
(p<0,001)\end{array}$ \\
\hline & & & & $418 \mu \mathrm{g}$ folato alimentar & Sem alteração \\
\hline $\begin{array}{l}\text { Venn et al. }{ }^{63} \\
\text { (caso-controle) }\end{array}$ & $\begin{array}{l}\text { Adultos e idosos } \\
\text { saudáveis }\end{array}$ & 34 & 17 & $628 \mu \mathrm{g}$ folato alimentar & $\begin{array}{l}\downarrow 12,0 \text { para } 9,7 \mu \mathrm{mol} / \mathrm{L} \\
\text { de Hct } \\
(\mathrm{p}<0,05)\end{array}$ \\
\hline \multirow[t]{2}{*}{$\begin{array}{l}\text { Venâncio et al. } \\
\text { (caso-controle) }\end{array}$} & Arterial periférica & 40 & 4 & $\begin{array}{l}500 \mu \mathrm{g} \text { de ácido fólico }+ \\
40 \mathrm{mg} \text { de cobalamina }\end{array}$ & $\begin{array}{l}\downarrow 39,6 \% \mathrm{Hct} \\
(\mathrm{p}<0,001)\end{array}$ \\
\hline & & & & $250 \mathrm{mg}$ de piridoxina & Sem alteração \\
\hline Pereira $^{65}$ (cruzado) & $\begin{array}{l}\text { Hipertensão } \\
\text { arterial }\end{array}$ & 69 & 8 & $500 \mu \mathrm{g}$ de ácido fólico & $\begin{array}{l}\downarrow 11 \text { a } 19 \% \text { Hct } \\
(\mathrm{p}<0,05)\end{array}$ \\
\hline
\end{tabular}

$\downarrow=$ redução; Hct = homocisteína plasmática.

ráveis do folato no processamento de alimentos em temperaturas elevadas. Desta forma, 50 a $90 \%$ do conteúdo dessa vitamina pode ser destruído pela cocção ou por outros processos tais como de envase e refinamento ${ }^{55}$.

O consumo de alimentos fortificados ou suplementos com vitamina B12 também é recomendado na hiper-homocisteinemia em idosos, pois 10 a 30\% desses pacientes podem apresentar má absorção dessa vitamina pela redução do fator intrínseco, e os suplementos garantem absor- ção adequada por difusão passiva, portanto sem a presença de fator intrínseco ${ }^{66}$.

Apesar da existência de estudos demonstrando que a ingestão de alimentos ricos em folato não foi suficiente para reduzir a homocisteína plasmática, outros estudos mostraram que, após a ingestão de aproximadamente $600 \mu \mathrm{g}$ de folato alimentar presente em alimentos de origem vegetal (hortaliças, legumes e frutas), houve redução da homocisteinemia, além de benefícios adicionais de re- 
dução no consumo de gordura saturada e aumento no consumo de fibras, ferro, tiamina, vitamina C e B6. Vale relatar que, ao contrário dos suplementos ou alimentos fortificados, a quantificação do consumo de folato alimentar é imprecisa devido à variação das tabelas de composição, e portanto necessita de diversos períodos de observação ${ }^{61,63}$.

Outros efeitos terapêuticos da utilização de vitaminas, em especial do folato, foram investigados na hiper-homocisteinemia, sobretudo em alguns marcadores biológicos do processo de aterosclerose possivelmente associados à hiper-homocisteinemia, como a disfunção endotelial, coagulabilidade, proliferação de células musculares, atividade de espécies reativas do metabolismo de oxigênio ${ }^{45}$, resposta pró-inflamatória ${ }^{67,68}$ e oxidação da fração $\operatorname{LDL}^{46}$. Nesse sentido, alguns estudos realizados na área de cardiologia $^{69-71}$ em indivíduos saudáveis ${ }^{72-74}$, adultos e idosos, hiper-homocisteinêmicos ou não, mostraram redução da disfunção endotelial pelo aumento significante da resposta vasodilatadora dependente ou independente do endotélio da artéria braquial com altas doses de ácido fólico (500 a $1.000 \mu \mathrm{g}$ ) em 6 a 12 semanas de tratamento. Nesses estudos, a avaliação da resposta vasodilatadora dependente do endotélio foi realizada por comparação em resposta a um período de isquemia (torniquete), e a resposta vasodilatadora independente do endotélio, ou seja, por ação direta sobre as células musculares lisas, foi avaliada após administração de agentes vasoativos como a nitroglicerina sublingual. Utilizando essas técnicas, foi possível analisar o diâmetro e as mudanças do fluxo sanguíneo na artéria braquial através do Doppler ultrassom e da pletismografia. $\mathrm{O}$ mecanismo pelo qual o ácido fólico melhora a resposta vasodilatadora é desconhecido, mas especula-se que, com a redução da homocisteína circulante haja uma maior biodisponibilidade de óxido nítrico pelo estímulo da óxido nítrico-sintase endotelial, o que favorece a vasodilatação ${ }^{73}$.

A utilização de ácido fólico por 2 meses em modelo experimental de ratos hiper-homocisteinêmicos submetidos a endarterectomia de carótida foi eficiente em reduzir a concentração de homocisteína e a hiperplasia intimal, avaliada através porcentagem de estenose luminal. Os achados desse estudo sustentam a teoria de que a hiper-homocisteinemia aumenta o estímulo mitogênico das células musculares lisas, produzindo uma ampla hiperplasia intimal em resposta ao modelo de endarterectomia de carótida. Com base nesses resultados, os autores sugeriram a análise da homocisteína plasmática no pré-operatório de endarterectomia: se for constatada a hiper-homocisteinemia, os pacientes poderiam iniciar a terapia com ácido fólico e evitar a reestenose pós-endarterectomia ${ }^{75}$. Em outro estudo experimental com ratos, a indução da hiper-homocisteinemia aumentou a porcentagem de lesão endotelial em vasos cerebrais visualizados por microscopia eletrônica; segundo os autores, a lesão foi provocada por alterações no metabolismo oxidativo intracelular. As lesões endoteliais encontradas nos ratos hiper-homocisteinêmicos eram semelhantes às lesões tipicamente encontradas em doenças cerebrais degenerativas como Alzheimer e Parkinson. Em contrapartida, a inclusão de doses moderadas de ácido fólico na dieta por 8 semanas resultou em mudanças morfológicas e, consequentemente, reduziu significantemente essas lesões endoteliais ${ }^{76}$. Em 250 pacientes submetidos a angioplastia coronariana, a terapia combinada de ácido fólico $(1.000 \mu \mathrm{g})$, vitamina B12 (400 $\mu \mathrm{g})$ e B6 (10 mg), utilizada por 6 meses, pôde ser utilizada como terapia adjuvante na angioplastia coronariana, pois promoveu redução significante da homocisteinemia e da taxa de reestenose pós-angioplastia ${ }^{77}$.

A influência da terapia com ácido fólico sobre marcadores inflamatórios sistêmicos da aterosclerose, como a PCR e a LDL-oxidada, ainda é controversa. Resultados observados em um recente estudo com homens e mulheres saudáveis que receberam $800 \mu \mathrm{g}$ de ácido fólico $(\mathrm{n}=264)$ ou placebo $(n=266)$ diariamente por 12 meses indicaram que houve aumento de $400 \%$ na concentração de folato sérico, redução significante nas concentrações de homocisteína (28\%) e manutenção das concentrações de PCR ultrassensível (PCR-us) e LDL-oxidada ${ }^{78}$. Em estudo controlado duplo-cego semelhante ao anterior, com 65 pacientes hiper-homocisteinêmicos portadores de DAP sintomática recebendo placebo ou combinação de vitaminas do complexo B (B1, B6 e B12) ou ácido fólico (500 $\mu \mathrm{g})$ por 6 meses, observou-se que o tratamento para redução da homocisteína não alterou os marcadores de homeostase (fator tissular, TF, e inibidor da via do fator tissular, TFPI), de inflamação crônica (como a PCR-us), interleucinas (6, 8 e $18)$ e proteína quimiotática de monócito (MCP-1 $)^{79}$. Os autores concluíram que o tratamento para redução da homocisteína em pacientes com DAP sintomática é questionável quanto ao controle do processo inflamatório crônico inerente à aterosclerose e interrogaram sobre o efeito da hi- 
per-homocisteinemia na inflamação crônica e na coagulação de pacientes com DAP sintomática. A utilização de vitaminas antioxidantes como o alfa-tocoferol, ácido ascórbico, beta-caroteno, associadas ao ácido fólico e à vitamina B12 por 15 dias, foi mais efetiva em reduzir significantemente a oxidação da LDL in vitro em pacientes com doença arterial coronariana ${ }^{80}$.

Tendo em vista o conjunto desses trabalhos, para se combater formas leves de hiper-homocisteinemia, a adequação dietética ou a utilização de alimentos fortificados com ácido fólico seria a maneira mais indicada; já nos casos de hiper-homocisteinemia moderada, a suplementação medicamentosa com ácido fólico seria a mais recomenda$\mathrm{da}$, por ser mais eficiente em reduzir a homocisteinemia.

\section{Efeitos do tratamento da hiper-homocisteinemia sobre a morbidade e mortalidade por doenças vasculares}

Atualmente, diversos estudos prospectivos foram realizados para avaliar os efeitos da suplementação com as vitaminas envolvidas no metabolismo da homocisteína sobre a prevalência e/ou a progressão das lesões ateroscleróticas nos diversos setores vasculares.

Em estudo pioneiro, 232 pacientes adultos portadores de DAP sintomática, sendo 30\% com hiper-homocisteinemia $(17,8 \pm 12,7 \mu \mathrm{mol} / \mathrm{L})$, foram tratados com 500 $\mu \mathrm{g}$ de ácido fólico associado à vitamina B6 (250 mg) por 2 anos. Houve redução significante da concentração de homocisteína $(17,8 \pm 12,7$ para $8,2 \pm 2,2 \mu \mathrm{mol} / \mathrm{L})$ e da frequência da progressão da DAP, mas não da incidência de novos eventos cerebrais ou coronarianos nos pacientes hiperhomocisteinêmicos após o tratamento vitamínico em comparação com os normo-homocisteinêmicos não tratados. Nesse estudo, os diagnósticos de progressão foram: 1) sintomatologia juntamente com a redução do índice de pressão tornozelo-braço e/ou por angiografia para DAP; 2) presença de ataque isquêmico transitório ou acidente vascular cerebral (AVC) para doença cerebral; 3) presença do infarto agudo do miocárdio ou progressão da angina pectoris para doença arterial coronariana ${ }^{81}$. Em outro estudo, realizado com pacientes com doença vascular periférica, coronariana ou cerebral, a suplementação com ácido fólico $(500 \mu \mathrm{g})$ associada à vitamina B6 $(250 \mathrm{mg})$, quando comparada com placebo, mostrou somente redução da taxa anormal do teste eletrocardiográfico no exercício, mas nenhum efeito sobre o índice de pressão tornozelo-braço ou sobre a regressão de lesões ateroscleróticas na carótida e artéria femoral $^{82}$.

Em concordância com o estudo anterior, a suplementação com $500 \mu \mathrm{g}$ de ácido fólico por 2 anos não reduziu a recorrência de eventos cardiovasculares em 300 pacien$\operatorname{tes}^{83}$.

Com o objetivo de verificar a recorrência de AVC, novos eventos coronarianos e morte, foi realizado um estudo multicêntrico duplo-cego randomizado controlado denominado VISP (The Vitamin Intervention for Stroke Prevention), que avaliou 3.680 adultos e idosos com diagnóstico prévio de AVC. Os participantes que apresentavam homocisteína plasmática abaixo de $10 \mu \mathrm{mol} / \mathrm{L}$ foram distribuídos em dois grupos distintos, um recebendo altas doses de ácido fólico, vitamina B6 e B12 (2.500 $\mu \mathrm{g}$, $25 \mathrm{mg}$ e $400 \mu \mathrm{g}$, respectivamente) e outro recebendo baixas doses (20 $\mu \mathrm{g}, 0,2 \mathrm{mg}$ e $6 \mu \mathrm{g}$, respectivamente). Após 2 anos de seguimento, a suplementação com baixas doses das vitaminas foi mais eficiente em reduzir a homocisteína, o risco de AVC $(10 \%, \mathrm{p}=0,05)$, de eventos coronarianos $(26 \%, \mathrm{p}<0,001)$ e de morte $(16 \%, \mathrm{p}=0,001)$. Uma provável explicação para o tratamento com altas doses não ter sido eficiente estaria associado à homocisteinemia no pré-tratamento, uma vez que a resposta ao tratamento vitamínico sabidamente é mais eficiente em indivíduos hiperhomocisteinêmicos ${ }^{84}$.

A fortificação de cereais com ácido fólico e o uso de suplementos reduziu o risco relativo de doença coronariana em 8 a 23\% em mulheres com idade acima de 55 anos e em 11 a $13 \%$ em homens com mais de $45 \operatorname{anos}^{85,86}$; de acordo com os autores, esta pode ser uma estratégia benéfica e não onerosa somente nos casos de hiper-homocisteinemia.

Diante dos resultados conflitantes desses estudos prospectivos, diversas revisões recentes concluíram que estudos bem desenhados deveriam ser realizados urgentemente para avaliar o impacto do tratamento da hiperhomocisteinemia sobre a morbimortalidade ${ }^{87,88}$ e progressão das doenças vasculares, incluindo a DAP ${ }^{89}$. Os autores dessas revisões enfatizaram que a homocisteína é fácil de ser avaliada e, mais importante, fácil e barata de ser tratada, muito embora alguns estudos clínicos interroguem os efeitos benéficos dessa terapêutica ${ }^{90}$. Por outro lado, o declínio da mortalidade cardiovascular nos Estados Unidos 
poderia ser atribuído, em parte, à fortificação com folato desde 1998, presumidamente pelo aumento do folato sanguíneo e pela redução das concentrações sanguíneas de homocisteína ${ }^{91}$.

\section{Conclusão}

As informações atualmente disponíveis evidenciam que a suplementação combinada de folato, vitamina B12 e vitamina B6 pode ser utilizada no tratamento da hiperhomocisteinemia. De fato, a suplementação, ou a fortificação, ou a adequação dietética isolada do folato promove redução eficiente da homocisteína plasmática e configura-se um procedimento custo-efetivo que apresenta eficácia, sem reações adversas, tratando-se, portanto, de um coadjuvante terapêutico com relação risco-benefício bastante favorável no controle da homocisteinemia. Quanto ao impacto da suplementação sobre a morbimortalidade por doenças vasculares, os estudos mostraram-se divergentes e inconclusivos.

\section{Referências}

1. Stoyioglou A, Jaff MR. Medical treatment of peripheral arterial disease: a comprehensive review. J Vasc Interv Radiol. 2004;15:1197-207.

2. Hiatt WR. Medical treatment of peripheral arterial disease and claudication. N Engl J Med. 2001;344:1608-21.

3. Lastória S, Maffei FHA. Aterosclerose obliterante periférica: epidemiologia, fisiopatologia, quadro clínico e diagnóstico. In: Maffei FHA, Lastória S, Yoshida WB, Rollo HA, editores. Doenças vasculares periféricas. Rio de Janeiro: Medsi; 2002. p. 1007-24.

4. Brasil, Ministério da Saúde [site na internet]. DATASUS. SIM - Sistema de Informação sobre Mortalidade. Brasília: Ministério da Saúde; 2007. [Citado 2008 fev 10]. http:// tabnet.datasus.gov.br/cgi/tabcgi.exe?sim/cnv/obtuf.def.

5. World Health Organization [site na internet]. Core health indicators 2002. [Citado 2008 mar 02]. http://www3.who.int/whosis/core/core_select_process.cfm.

6. Bhatt DL, Steg PG, Ohman EM, et al. International prevalence, recognition, and treatment of cardiovascular risk factors in outpatients with atherothrombosis. JAMA. 2006;295:180-9.

7. Norgren L, Hiatt WR, Dormandy J, Nehler M, Harris K, Fowkes F. Inter-society consensus for the management of peripheral arterial disease (TASC II). Eur J Vasc Endovasc Surg. 2007;33:S1-75.

8. Hirch AT, Criqui MH, Treat-Jacobson D, et al. Peripheral arterial disease detection, awareness, and treatment in primary care. JAMA. 2001;286:1317-24.

9. Nygard O, Vollset SE, Refsum H, Brattström L, Ueland PM. Total homocysteine and cardiovascular disease. J Intern Med. 1999;246:425-54.
10. McCully KS. Vascular pathology of homocysteinemia: implications for the pathogenesis of arteriosclerosis. Am J Pathol. 1969;56:111-28.

11. Malinow MR. Homocyst(e)ine and arterial occlusive diseases. J Intern Med. 1994;236:603-17.

12. Rassoul F, Richter V, Janke C, et al. Plasma homocysteine and lipoprotein profile in patients with peripheral arterial occlusive disease. Angiology. 2000;51:189-96.

13. Selhub J. Homocysteine metabolism. Annu Rev Nutr. 1999;19:217-46.

14. Selhub J, Miller JW. The pathogenesis of homocysteinemia: interruption of the coordinate regulation by S-adenosylmethionine of the remethylation and transulfuration of homocysteine. Am J Clin Nutr. 1992;55:131-8.

15. Ueland PM, Refsum H, Stabler SP, Malinow MR, Andersson A, Allen RH. Total homocysteine in plasma or serum: methods and clinical applications. Clin Chem. 1993;39:1764-79.

16. Kang SS, Wong PWK, Malinow MR. Hyperhomocys$\mathrm{t}(\mathrm{e})$ inemia as a risk factor for occlusive vascular disease. Annu Rev Nutr. 1992;12:279-98.

17. Boushey CJ, Beresford SAA, Omenn GS, Motulsky AG. A quantitative assessment of plasma homocysteine as a risk factor for vascular disease. JAMA. 1995;274:1049-57.

18. Venancio LS. Indicadores nutricionais e níveis de homocisteína em pacientes com doença arterial periférica [dissertação]. Botucatu: Universidade Estadual Paulista; 2002.

19. Mölgaard J, Malinow MR, Lassvik C, Holm AC, Upson B, Olsson AG. Hyperhomocyst(e)inaemia: an independent risk factor for intermittent claudication. J Intern Med. 1992;231:273-9.

20. Malinow MR, Kang SS, Taylor LM, et al. Prevalence of hyperhomocyst(e)inemia in patients with peripheral arterial occlusive disease. Circulation. 1989;79:1180-8.

21. Brattström L, Israelson B, Norrving B, et al. Impaired homocysteine metabolism in early-onset cerebral and peripheral occlusive arterial disease. Atherosclerosis. 1990;81:51-60.

22. Tsai MY, Arnett DK, Eckfeldt JH, Willians RR, Ellison RC. Plasma homocysteine and its association with carotid intimal-medial wall thickness and prevalent coronary heart disease. Atherosclerosis. 2000;151:519-24.

23. Brunelli T, Prisco D, Fedi S, et al. High prevalence of mild hyperhomocysteinemia in patients with abdominal aortic aneurysm. J Vasc Surg. 2000;32:531-6.

24. Tavares JR, D'Almeida V, Diniz DC, et al. Analysis of plasma homocysteine levels in patients with unstable angina. Arq Bras Cardiol. 2002;79:167-72.

25. Taddei CFG, Batlouni M, Sarteschi C, et al. Hiper-homocisteinemia como fator de risco para doença aterosclerótica coronariana em idosos. Arq Bras Cardiol. 2005;85:166-73.

26. Faria-Neto JR, Chagas ACP, Bydlowski SP, et al. Hyperhomocysteinemia in patients with coronary artery disease. Braz J Med Biol Res. 2006;39:435-63.

27. Garófolo L, Barros Junior N, Ferreira SRG, Sanudo A, Miranda Junior F. Moderate hyperhomocysteinemia in atherosclerotic peripheral arterial disease in the diabetics Nipo-Brazilian population in the city of Bauru. J Vasc Bras. 2003;2(Suppl 1):S82.

28. Miranda Junior F, Garófolo L, Barros Junior N, Ferreira SRG, Sanudo A. Influence of gender in a association be- 
tween moderate hyperhomocysteinemia and peripheral arterial disease in diabetics Nipo-Brazilian in the city of Bauru. $\mathrm{J}$ Vasc Bras 2003;2(Suppl 1):S82.

29. Graham IM, Daly LE, Refsum H, et al. Homocisteína plasmática como fator de risco para doença cardiovascular. JAMA. 1997;277:1775-81.

30. Nicoloff AD, Taylor LM, Sexton GJ, et al. Relationship between site of initial symptoms and subsequent progression of disease in a prospective study of atherosclerosis progression in patients receiving long-term treatment for symptomatic peripheral arterial disease. J Vasc Surg. 2002;35:38-47.

31. Taylor LM, De Frang RD, Harris Junior EJ, Porter JM. The association of elevated plasma homocyst(e)ine with progression of symptomatic peripheral arterial disease. J Vasc Surg. 1991;13:128-36.

32. Taylor LM, Moneta GL, Sexton GJ, Schuff RA, Porter JM. Prospective blinded study of the relationship between plasma homocysteine and progression of symptomatic peripheral arterial disease. J Vasc Surg. 1999;29:8-21.

33. Selhub J, Jacques PF, Wilson PWF, Rush D, Rosenberg IH. Vitamin status and intake as primary determinants of homocysteinemia in an elderly population. JAMA. 1993;270:2693-8.

34. Cattaneo M. Hyperhomocysteinemia, atherosclerosis and thrombosis. Thromb Haemost. 1999;81:165-76.

35. Frosst P, Blom HJ, Milos R, et al. A candidate genetic risk factor for vascular disease: a common mutation in methylenetetrahydrofolate reductase. Nat Genet. 1995;10:111-3.

36. Verhoeff BJ, Trip MD, Prins MH, Kastelein JJP, Reitsma $\mathrm{PH}$. The effect of a common methylenetetrahydrofolate reductase mutation on levels of homocysteine, folate, vitamin B12 and on the risk of premature atherosclerosis. Atherosclerosis. 1998;141:161-6.

37. Arruda VR, Von Zuben PM, Chiaparini LC, AnnichinoBizzacchi JM, Costa FF. The mutation Ala $677{ }^{\circledR}$ Val in the methylenetetrahydrofolate reductase gene: a risk factor for arterial disease and venous thrombosis. Thromb Haemost. 1997;77:818-21.

38. Muniz MTC. Avaliação do polimorfismo genético para MTHFR (metilenotetrahidrofolato redutase) e sua correlação com os níveis de homocisteína em pacientes com doença arterial coronariana [dissertação]. Recife: Universidade Federal de São Paulo; 2002.

39. Jacques PF, Bostom AG, Williams RR, et al. Relation between folate status, a common mutation in methylenetetrahydrofolate reductase, and plasma homocysteine concentrations. Circulation. 1996;93:7-9.

40. Harker LA, Slichter SJ, Scott CR, Ross R. Homocysteinemia, vascular injury and arterial thrombosis. N Engl J Med. 1974;291:537-43.

41. Harker LA, Ross R, Slichter SJ, Scott CR. Homocysteine-induced arteriosclerosis. The role of endothelial cell injury and platelet response in its genesis. J Clin Invest. 1976;58:731-41.

42. França LHG, Pereira AH, Perini SC, et al. Aterogênese em artéria ilíaca comum de suínos submetidos à homocisteinemia induzida pela ingestão de metionina. J Vasc Bras. 2006;5:11-6.
43. Stahlke Junior HJ, França LHG, Stahlke PH, Stalhke PS. Hiper-homocisteinemia causando aterogênese na aorta de coelhos-modelo experimental. J Vasc Bras. 2004;3:20-30.

44. Cerqueira NF, Yoshida WB. Óxido nítrico. Revisão. Acta Cir Bras. 2002; 17:417-23.

45. Lentz SR. Mechanisms of homocysteine-induced atherothrombosis. J Thromb Haemost. 2005;8:1646-54.

46. Blom HJ. Consequences of homocysteine export and oxidation in the vascular system. Semin Thromb Haemost. 2000;26:227-32.

47. Austin RC, Lentz SR, Werstuck GH. Role of hyperhomocysteinemia in endothelial dysfunction and atherothrombotic disease. Cell Death Diff. 2004;11:S56-64.

48. Poddar R, Sivasubramanian N, Dibello PM, Robinson K, Jacobsen DW. Homocysteine induces expression and secretion of monocyte chemoattractant protein-1 and interleukin-8 in human aortic endothelial cells. Implications for vascular disease. Circulation. 2001;103:2717-23.

49. Welch GN, Loscalzo J. Homocysteine and atherothrombosis. N Engl J Med. 1998;338:1042-9.

50. Graham IM, O'Callaghan P. Vitamins, homocysteine and cardiovascular risk. Cardiovasc Drugs Ther. 2002;16:383-9.

51. Selhub J, Jacques PF, Wilson PWF, Rush D, Rosenberg IH. Vitamin status and intake as primary determinants of homocysteinemia in an elderly population. JAMA. 1993;270:2693-8.

52. Durand P, Prost M, Loreau N, Lussier-Cacan A, Blache D. Impaired homocysteine metabolism and atherothrombotic disease. Lab Invest. 2001;81:645-72.

53. Merchant AT, Hu FB, Spiegelman D, Willet WC, Rimm EB, Ascherio A. The use of B vitamin supplements and peripheral arterial disease risk in men are inversely related. J Nutr. 2003;133:2863-7.

54. US Department of Agriculture, Agricultural Research Service [site na internet]. USDA Nutrient Database for Standard Reference, Release 11. Nutrient Data Laboratory Home Page, http://www.nal.usda.gov/fnic/foodcomp.

55. Institute of Medicine, Food and Nutrition Board. Dietary Reference Intakes (DRI) for thiamin, riboflavin, niacin, vitamin B6, folate, vitamin B12, pantothenic acid, biotin, and coline. Washington: National Academy Press; 2000.

56. Jacques PF, Selhub J, Bostom AG, Wilson PWF, Rosenberg IH. The effect of folic acid fortification on plasma folate and total homocysteine concentrations. N Engl J Med. 1999;340:1449-54.

57. Malinow MR, Duell PB, Hess DL, et al. Reduction of plasma homocyst(e)ine levels by breakfast cereal fortified with folic acid in patients with coronary heart disease. N Engl J Med. 1998;338:1009-15.

58. Brasil, Ministério da Saúde. Guia alimentar para a população brasileira: promovendo a alimentação saudável. Referencial teórico - Deficiências nutricionais. Brasília: Ministério da Saúde; 2005. p. 20.

59. Brasil, Ministério da Saúde, Agência Nacional de Vigilância Sanitária [site na internet]. Farinha terá ácido fólico para combater anencefalia em bebês. Brasília: ANVISA; 2002. [citado: 2006 jul 23]. http://www.anvisa.gov.br/divulga/informes/2002. 
60. Homocysteine Lowering Trialists' Collaboration. Lowering blood homocysteine with folic acid based supplements: meta-analysis of randomized trials. BMJ. 1998;316:894-8.

61. Brouwer IA, van Dusseldorp M, West CE, et al. Dietary folate from vegetables and citrus fruit decreases plasma homocysteine concentrations in humans in a dietary controlled trial. J Nutr. 1999;129:1135-9.

62. Riddell LI, Chisholm A, Williama S, Mann JI. Dietary strategies for lowering homocysteine concentrations. Am J Clin Nutr. 2000;71:1448-54.

63. Venn BJ, Mann JI, Williams SM, et al. Dietary counseling to increase natural folate intake: a randomized, placebocontrolled trial in free-living subjects to assess effects on serum folate and plasma total homocysteine. Am J Clin Nutr. 2002;76:758-65.

64. Venâncio LS, Santos MDB, Yoshida WB. Efeitos da suplementação vitamínica de ácido fólico, vitamina B12 e vitamina B6 sobre a concentração de homocisteína em indivíduos portadores de doença arterial periférica. J Vasc Bras. 2003;2(Suppl 1):S83.

65. Pereira AF. Efeito da suplementação de ácido fólico e do exercício físico sobre as concentrações de homocisteína plasmática em indivíduos portadores de hipertensão arterial essencial [dissertação]. São Paulo: Universidade de São Paulo; 2004.

66. Omenn GS, Beresford SAA, Motulsky AG. Preventing coronary heart disease: B vitamins and homocysteine. Circulation. 1998;97:421-4.

67. Kuller LH, Evans RW. Homocysteine, vitamins and cardiovascular disease. Circulation. 1998;98:196-9.

68. Brevetti G, Silvestro A, Di Giacomo S, et al. Endothelial dysfunction in peripheral arterial disease is related to increase in plasma markers of inflammation and severity of peripheral circulatory impairment but not to classic risk factors and atherosclerotic burden. J Vasc Surg. 2003;38:374-9.

69. Chambers JC, Ueland PM, Obeid OA, Wrigley J, Refsum H, Kooner JS. Improved vascular endothelial function after oral $B$ vitamins: an effect mediated through reduced concentrations of free plasma homocysteine. Circulation. 2000;120:2479-83.

70. Title LM, Cummings PM, Giddens K, Genest JJ, Nassar BA. Effect of folic acid and antioxidant vitamins on endothelial dysfunction in patients with coronary artery disease. J Am Coll Cardiol. 2000;36:758-65.

71. Thambyrajah J, Landray MJ, Jones HJ, Mcglynn FJ, Wheeler DD, Townend JN. A randomized double-blind placebocontrolled trial of the effect of homocysteine-lowering therapy with folic acid on endothelial function in patients with coronary artery disease. J Am Coll Cardiol. 2001;37:1858-63.

72. Bellamy MF, Mcdowell IFW, Ramsey NM, Brownlee M, Newcombe RG, Lewis MJ. Oral folate enhances endothelial function in hyperhomocystaemic subjects. Eur J Clin Invest. 1999;29:659-62.

73. Usui M, Matsuoka H, Miyzaki H, Ueda S, Okuda S, Imaizumi T. Endothelial dysfunction by acute hyperhomocyst(e)inaemia: restoration by folic acid. Clin Sci. 1999;96:235-9.
74. Woo KS, Chook P, Lolin YI, Sanderson JE, Metreweli C, Celermajer DS. Folic acid improves arterial endothelial function in adults with hyperhomocysteinemia. J Am Coll Cardiol. 1999;34:2002-6.

75. Smith TP, Cruz CP, Brown AT, Eidt JF, Moursi MM. Folate supplementation inhibits intimal hyperplasia induced by a high-homocysteine diet in a rat carotid endarterectomy model. J Vasc Surg. 2001;34:474-81.

76. Lee H, Kim J, Kim HJ, Lee I, Chang N. Folic acid supplementation can reduce the endothelial damage in rat brain microvasculature due to hyperhomocysteinemia. J Nutr. 2005;135:544-8.

77. Schnyder G, Roffi M, Pin R, et al. Decreased rate of coronary restenosis after lowering of plasma homocysteine levels. $\mathrm{N}$ Engl J Med. 2001;345:1593-600.

78. Durga J, van Tits LJH, Schooten EG, et al. Effect of lowering of homocysteine levels on inflammatory markers. A randomized controlled trial. Arch Intern Med. 2005;165:1388-94.

79. Schernthaner GH, Plank C, Minar E, et al. No effect of homocysteine-lowering therapy on vascular inflammation and haemostasis in peripheral arterial occlusive disease. Eur $\mathrm{j}$ Clin Invest. 2006;36:333-9.

80. Bunout D, Garrido A, Suazo M, et al. Effects of supplementation with folic acid and antioxidant vitamins on homocysteine levels and LDL oxidation in coronary patients. Nutrition. 2000;16:107-110.

81. De Jong SC, Stehouwer CDA, Van Den Berg M, Geurts TW, Bouter LM, Rauwerda JA. Normohomocysteinaemia and vitamin-treated hyperhomocysteinaemia are associated similar risks of cardiovascular events in patients with premature peripheral arterial occlusive disease. A prospective cohort study. J Intern Med. 1999;246:87-96.

82. Vermeulen EGJ, Stehouwer CDA, Twisk JWR, et al. Effect of homocysteine-lowering treatment with folic acid plus vitamin B6 on progression of subclinical atherosclerosis: a randomized, placebo-controlled trial. Lancet. 2000;355:517-22.

83. Liem A, Reynierse-Buitenwerf GH, Zwinderman AH, Jukema JW, van Veldhuisen DJ. Secondary prevention with folic acid: effects on clinical outcomes. J Am Coll Cardiol. 2003:41:2105-13.

84. Toole JF, Malinow MR, Chambless LE, et al. Lowering homocysteine in patients with ischemic stroke to prevent recurrent stroke, myocardial infarction, and death. The vitamins intervention for stroke prevention (VISP) randomized controlled trial. JAMA. 2004;291:565-75.

85. Nallamothu BK, Fendrick AM, Rubenfire M, Saint S, Bandekar RR, Omenn GS. Potential clinical and economic effects of homocysteine lowering. Arch Intern Med. 2000;160:3406-12.

86. Tice JA, Ross E, Coxson PG, et al. Cost-effectiveness of vitamin therapy to lower plasma homocysteine levels for the prevention of coronary heart disease. Effect of grain fortification and beyond. JAMA. 2001;286:936-43.

87. Lonn E. Homocysteine in the prevention of ischemic heart disease, stroke and venous thromboembolism: therapeutic target or just another distraction? . 2007;14:481-7.

88. Wierzbicki AS. Homocysteine and cardiovascular disease: a review of the evidence. Diab Vasc Dis Res. 2007;4:143-50. 
89. Hansrani M, Stansby G. Homocysteine lowering interventions for peripheral arterial disease and bypass grafts. Cochrane Database Syst Rev. 2002;(3):CD003285.

90. Khandanpour N, Loke YK, Meyer FJ, Jennings B, Armon MP. Eur J Vasc Endovasc Surg. 2009;38:316-22.

91. McCully KS. Homocysteine, vitamins, and vascular disease prevention. Am J Clin Nutr. 2007;86:1563S-8S.

\section{Correspondência:}

Luciene de Souza Venâncio

Floriano Peixoto, 240 - Centro

Caixa Postal 334

18603-970 - Botucatu, SP

Tel.: (14) 3882.6570

E-mail: lucienenutri@yahoo.com.br

\section{Contribuições dos autores}

Concepção e desenho do estudo: LSV, RCB, WBY

Análise e interpretação dos dados: LSV, WBY

Coleta de dados: N/A

Redação do artigo: LSV, WBY

Revisão crítica do texto: LSV, WBY

Aprovação final do artigo*: LSV, RCB, WBY

Análise estatística: N/A

Responsabilidade geral pelo estudo: LSV

Informações sobre financiamento: CAPES e FAPESP

* Todos os autores leram a aprovaram a versão final submetida ao J Vasc Bras. 